\title{
ON REFLECTION LENGTH IN REFLECTION GROUPS
}

\author{
R.B. Howlett AND G.I. LeHRER \\ School of Mathematics and Statistics \\ University of Sydney \\ N.S.W. 2006, Australia \\ Centre for Mathematics and its Applications \\ Australian National University \\ A.C.T. 0200, Australia
}

\begin{abstract}
Let $W$ be the Weyl group of a connected reductive group over a finite field. It is a consequence of the Borel-Tits rational conjugacy theorem for maximal split tori that for certain reflection subgroups $W_{1}$ of $W$ (including all parabolic subgroups), the elements of minimal reflection length in any coset $w W_{1}$ are all conjugate, provided $w$ normalises $W_{1}$. We prove a sharper and more general result of this nature for any finite Coxeter group. Applications include a fusion result for cosets of reflection subgroups and the counting of rational orbits of a given type in reductive Lie algebras over finite fields.
\end{abstract}

\section{Background And Statement of RESUlts}

Let $W$ be a finite Coxeter group acting as a reflection group on the Euclidean space $V$ of dimension $\ell$. We refer to [B] for background.

(1.1) Definition. The reflection length $n(w)$ of an element $w \in W$ is the minimal integer $n$ such that $w=r_{1} r_{2} \ldots r_{n}$, where the $r_{i}$ are reflections in $W$.

The function $n(w)$ is clearly conjugacy invariant and it is well known (see $[\mathrm{C} 1$, Lemma 2] or [HL], for example) that for any element $w \in W$, we have

$$
n(w)=\operatorname{dim}(\operatorname{im}(1-w)) .
$$

When $W$ is the Weyl group of a connected reductive group $G$ which is defined and split (see $[\mathrm{BT}]$ ) over a finite field $\mathbb{F}_{q}$, the function $n(w)$ arises in the study of rationality properties of tori. For background about the following matters the reader is referred to $[\mathrm{L}]$ and the references there. Let $F: G \rightarrow G$ be the Frobenius endomorphism associated with the $\mathbb{F}_{q}$-structure on $G$. We refer to an $F$-stable subvariety of $G$ as rational and denote by $H^{F}$ the set of $F$-fixed points of any variety $H$ on which $F$ acts. It is well-known that the $G^{F}$-conjugacy classes of rational (that is, $F$-stable) maximal tori of $G$ are parametrised by the conjugacy classes of $W$. Denote by $T_{0}$ a fixed maximal torus of $G$ which is split over $\mathbb{F}_{q}$. For any group $H$

1991 Mathematics Subject Classification. 20G40, 20 G05.

Typeset by $\mathcal{A}_{\mathcal{M S}}$-TEX 
defined over $\mathbb{F}_{q}$, denote by $r(H)$ its $\mathbb{F}_{q}$-rank (the dimension of any of its maximal

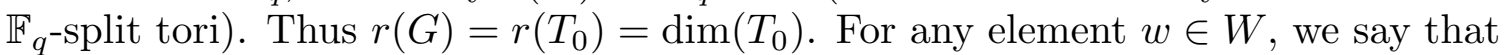
the rational maximal torus $T$ is $w$-twisted and write $T=T_{w}$ if $T=g T_{0} g^{-1}$ for some $g \in G$ such that $g^{-1} F(g)=\dot{w} \in N_{G}\left(T_{0}\right)$ where $\dot{w} T_{0}=w \in W=N_{G}\left(T_{0}\right) / T_{0}$. The set $\{w \in W \mid T$ is $w$-twisted $\}$ is a conjugacy class of $W$.

If $T_{w}$ is a $w$-twisted rational maximal torus, then we have (see $[\mathrm{L},(5.5)]$ )

$$
r\left(T_{w}\right)=\ell-n(w)
$$

where $\ell=r(G)$. Now suppose $x$ is a semisimple element of $G^{F}$. The connected centraliser $C_{G}(x)^{\circ}$ is a reductive group defined over $\mathbb{F}_{q}$ and the rational conjugacy of the maximally split maximal tori of $C_{G}(x)^{\circ}$, proved in $[\mathrm{BT}]$, has the following simple (but not obvious) consequence for the length function.

(1.4) Proposition. Let $W$ be the Weyl group of a connected reductive group $G$ over $\mathbb{F}_{q}$. Suppose $W_{1} \leq W$ is the Weyl group of the connected centraliser of a semisimple element of $G^{F}$ (e.g. $W_{1}$ could be any parabolic subgroup of $W-$ see $§ 2$ below). Let $w$ be an element of the normaliser in $W$ of $W_{1}$. Then any two elements of the coset $w W_{1}$ which have minimal reflection length in the coset are conjugate in $W$.

We shall explain how (1.4) follows from the work of Borel and Tits in the next section, but our main purpose in this note is to prove an elementary but more general result concerning reflection groups, of which (1.4) is a consequence.

(1.5) Theorem. Let $W$ be a finite Coxeter group acting on a Euclidean space $V$. Let $\Phi$ be the corresponding root system, with $\Pi$ a chosen base of $\Phi$. Let $\sigma$ be an orthogonal transformation of $V$ such that $\sigma \Pi=\Pi$. Then, for any element $w \in W$, the following conditions are equivalent

(1) $\operatorname{dim}(\operatorname{im}(1-\sigma w))$ is minimal

(2) There is an element $x \in W$ such that $\sigma w=x^{-1} \sigma x$.

(3) $\sigma w$ stabililises some simple system in $\Phi$.

We shall see below (see $\S 2$ or (4.4)(1)) that (1.4) follows easily from (1.5), with $W$ (of (1.5)) replaced by $W_{1}$. Moreover, (1.5) shows that the elements of minimal reflection length in $w W_{1}$ are actually conjugate by an element of $W_{1}$. If $W_{1}$ is any reflection subgroup of $W$, the choice of a simple system $\Pi$ for $W$ determines a length function for $W$ and it is the case (see $\S 4$ below) that each coset $w W_{1}$ contains a unique shortest element with respect to this length function. If $w$ normalises $W_{1}$, our theorem identifies the conjugacy class of elements of minimal reflection length in $w W_{1}$ as that of this shortest element in the coset (see (4.2) below).

\section{RATiONAL TORI}

In this section we indicate how (1.4) is related to the results of [BT]. We maintain the notation of $\S 1$ and assume that the derived group $G^{\prime}$ is simply connected. By a theorem of Steinberg [St $\S 8$ ] this implies that for any element $t \in T_{0}$, the centraliser $C_{W}(t)$ is a reflection subgroup of $W$. It is the case that all parabolic subgroups of $W$ arise in this way, as may be seen from the results in [C2], or from the fact that the corresponding stabilisers in the Lie algebra case are all the parabolic subgroups of $W$. 
Let $x$ be a semisimple element of $G^{F}$. If $T$ is any rational maximal torus in $C_{G}(x)^{\circ}$, then there exists an element $g \in G$ such that $T=g T_{0} g^{-1}$, and $g^{-1} F(g)$ is a representative in $N_{G}\left(T_{0}\right)$ of $w \in W=N_{G}\left(T_{0}\right) / T_{0}$. Moreover $x \in T$, so that $y=g^{-1} x g \in T_{0}$. Recall that since $T_{0}$ is split over $\mathbb{F}_{q}$, the $F$-action on $W$ is trivial.

(2.1) Proposition. (cf. $[L,(5.5)])$ Maintaining the notation above, let $W_{1}$ be the centraliser in $W$ of $y$. Then $W_{1}$ is a reflection subgroup of $W$ (see the remarks above) and we have

(1) The $W$-orbit of $y \in T_{0}$ is determined by $x$, independently of the choice of $T$ or $g$.

(2) $y$ is fixed by the endomorphism $w \circ F$ of $T_{0}$ (given by $t \mapsto w F(t) w^{-1}$ ).

(3) $w$ normalises $W_{1}$.

(4) $\left\{v \in W \mid y \in T_{0}^{v \circ F}\right\}=w W_{1}$.

Proof. The first part follows because any two elements of $T_{0}$ which are conjugate in $G$ are conjugate by an element of $W$, while the second part is a simple computation. For the third part, observe that since $F(y)=w^{-1} y w$,

$$
w^{-1} W_{1} w=C_{W}\left(w^{-1} y w\right)=C_{W}(F(y)) \supseteq F\left(C_{W}(y)\right)=F\left(W_{1}\right)=W_{1}
$$

The fourth part follows from the second, since $(v \circ F)(y)=(w \circ F)(y)$ if and only if $w^{-1} v \in C_{W}(F(y))=W_{1}$.

(2.2) Corollary. The coset $w W_{1} \subseteq W$ of (2.1) is determined up to conjugacy in $W$ by $x$.

This follows immediately from (2.1).

(2.3) Proposition. Maintain the above notation. The subset $S_{1}$ of $W$ consisting of those elements $v$ such that $C_{G}(x)^{\circ}$ contains a $v$-twisted rational maximal torus of $G$ coincides with the union $S_{2}$ of those conjugacy classes which are represented in $w W_{1}$.

Proof. Suppose $v \in S_{1}$. Then $x \in h T_{0} h^{-1}$ for some $h \in G$ with $h^{-1} F(h) \in v T_{0}$. By (2.2), $v$ is conjugate to an element of $w W_{1}$; hence $v \in S_{2}$. Conversely, let $u \in W_{1}$. To complete the proof we show that there is a $w u$-twisted maximal torus which contains $x$.

Since $C_{G}(y)^{\circ}$ has Weyl group $W_{1}$ we may choose $\dot{u} \in F\left(C_{G}(y)^{\circ}\right)$ with $\dot{u} T_{0}=u$. Now $F(g) \dot{u} F(g)^{-1} \in F\left(g C_{G}(y)^{\circ} g^{-1}\right)=F\left(C_{G}(x)^{\circ}\right) \subseteq C_{G}(x)^{\circ}$, and by Lang's Theorem we may choose $f \in C_{G}(x)^{\circ}$ with $f^{-1} F(f)=F(g) \dot{u} F(g)^{-1}$. Writing $k=f g$ and $T_{1}=k T_{0} k^{-1}$, we have $x=f x f^{-1}=k y k^{-1} \in T_{1}$, so that $T_{1} \subseteq C_{G}(x)^{\circ}$. Moreover,

$$
k^{-1} F(k)=g^{-1} f^{-1} F(f) F(g)=g^{-1} F(g) \dot{u}=\dot{w} \dot{u},
$$

which shows that $T_{1}$ is rational and wu-twisted.

We are now able to give the

Proof of (1.4). Given a subgroup $W_{1}$ of $W$ as specified in the statement of (1.4) and an element $w \in N_{W}\left(W_{1}\right)$, there is an element $y \in T_{0}$ with centraliser $W_{1}$ in $W$ and, provided $q$ is sufficiently large, which we may assume, $y \in T_{0}^{w \circ F}$. Let $g \in G$ satisfy $g^{-1} F(g)=\dot{w}$. Then let $x=g y g^{-1}$. By [BT, Theorème 4.21], the maximally split tori in $C_{G}(x)^{\circ}$ are conjugate in $C_{G}(x)^{\circ} F$ and hence a fortiori in $G^{F}$. But, by (1.3) 
and (2.3), the $G^{F}$-conjugacy classes of these correspond to the conjugacy classes of $W$ which are represented in $w W_{1}$ and have minimal reflection length among those classes. Hence there is a unique such class.

\section{Proof of the MaIn theorem}

We shall require the following two elementary results.

(3.1) Lemma. Let $V$ be a finite dimensional vector space over a field, equipped with a non-degenerate symmetric bilinear form (, ). If $\theta$ is any isometry of $V$, then $\operatorname{im}(1-\theta)=\operatorname{ker}(1-\theta)^{\perp}$, where $S^{\perp}$ denotes the perpendicular subspace of the subset $S$ of $V$.

The proof is easy and left to the reader. The second result is

(3.2) Proposition. Let $V$ be as in (3.1) and suppose $g(\neq 1)$ is an isometry of $V$. Assume the characteristic of the ground field is not two. Let a be a nonisotropic vector in $\mathrm{im}(1-g)$ and let $r_{a}$ be the involutory reflection in the hyperplane orthogonal to a. Then

$$
\operatorname{dim}\left(\operatorname{im}\left(1-r_{a} g\right)\right)=\operatorname{dim}(\operatorname{im}(1-g))-1
$$

Proof. By hypothesis, there is an element $v \in V$ such that $a=(1-g) v$. Then

$$
0 \neq(a, a)=(v-g v, v-g v)=2(v, v)-2(v, g v)
$$

Moreover

$$
\begin{aligned}
\left(1-r_{a} g\right) v & =v-r_{a}(g v) \\
& =v-g v+2 \frac{(g v, a)}{(a, a)} a \\
& =a+2\left(\frac{(g v, v)}{(a, a)}-\frac{(g v, g v)}{(a, a)}\right) a \\
& =a-a \\
& =0
\end{aligned}
$$

It follows that $g v=r_{a} v$, whence $\left(1-r_{a} g\right) a=0$. Now

$$
\operatorname{im}\left(1-r_{a} g\right)=\operatorname{im}\left(\left(1-r_{a}\right)+r_{a}(1-g)\right) \subseteq r_{a} \operatorname{im}(1-g)=\operatorname{im}(1-g)
$$

since $\operatorname{im}\left(1-r_{a}\right)=\operatorname{span}\{a\} \subseteq \operatorname{im}(1-g)$. But by $(3.1), a \notin \operatorname{im}\left(1-r_{a} g\right)$, since $a \in \operatorname{ker}\left(1-r_{a} g\right)$ and $a$ is not isotropic. Thus $a$ is a non-zero vector which is in $\operatorname{im}(1-g)$, but is not in $\operatorname{im}\left(1-r_{a} g\right)$. It follows that $\operatorname{dim}\left(\operatorname{im}\left(1-r_{a} g\right)\right)<\operatorname{dim}(\operatorname{im}(1-g))$.

On the other hand $\left(1-r_{a} g\right)=r_{a}\left(r_{a}-g\right)$ and since $r_{a}$ is a reflection, the rank of $\left(r_{a}-g\right)$ differs from that of $(1-g)$ by at most 1 . Hence the result.

We are now in a position to give the

Proof of (1.5). The equivalence of conditions (2) and (3) is clear from the transitivity of the action of $W$ on the set of simple systems. Suppose we know that (1) implies (2). Then it follows that the smallest value of $\operatorname{dim}(\operatorname{im}(1-\sigma w))$ is 
$\operatorname{dim}(\operatorname{im}(1-\sigma))$, whence $(2)$ implies (1). Thus the proof is reduced to showing that (1) implies (2).

Assume $w \in W$ is such that $\operatorname{dim}(\operatorname{im}(1-\sigma w))$ is minimal. By hypothesis, $\sigma$ permutes the elements of $\Pi$, whence conjugation by $\sigma$ permutes the generators of $W$. So $\sigma$ normalises $W$.

Let $D=\{v \in V \mid(v, a) \geq 0$ for all $a \in \Pi\}$ and recall that $V=\bigcup_{t \in W} t D$. Write $K=\operatorname{ker}(1-\sigma w)$. We wish to show that $\sigma w$ is conjugate to $\sigma$ by an element of $W$. Now since a real vector space is not the union of a finite number of proper subspaces, there is an element $t \in W$ such that $t^{-1} D \cap K$ spans $K$. If we write $w_{1}=\sigma^{-1} t \sigma w t^{-1} \in W$, then $\sigma w_{1}=t(\sigma w) t^{-1}$, so that $K_{1}=\operatorname{ker}\left(1-\sigma w_{1}\right)=t K$. Moreover $D \cap K_{1}$ spans $K_{1}$. We shall show that $w_{1}=1$.

If $w_{1} \neq 1$, there exists $a \in \Pi$ such that $w_{1}^{-1} a \in \Phi^{-}$. Write $b=\sigma a \in \Pi$. Then for all $v \in D \cap K_{1}$, we have

$$
0 \leq(b, v)=\left(b,\left(\sigma w_{1}\right) v\right)=\left(\left(w_{1}^{-1} \sigma^{-1}\right) b, v\right)=\left(w_{1}^{-1} a, v\right) \leq 0 .
$$

Hence $b \in\left(D \cap K_{1}\right)^{\perp}=K_{1}^{\perp}=\operatorname{im}\left(1-\sigma w_{1}\right)$ (by (3.1)). It follows from (3.2) that

$$
\operatorname{dim}\left(\operatorname{im}\left(1-r_{b} \sigma w_{1}\right)\right)<\operatorname{dim}\left(\operatorname{im}\left(1-\sigma w_{1}\right)\right),
$$

contradicting the minimality of the right hand side. Hence $w_{1}=1$, so that $\sigma w=t^{-1} \sigma t$, which completes the proof of the theorem.

\section{Applichtion to Reflection subgroups}

Suppose that $W$ is a finite Coxeter group on $V$, that $\Phi$ is its root system in $V$ and that $\Phi^{+}$and $\Pi$ are corresponding sets of positive and simple roots in $\Phi$. Let $W_{1}$ be any reflection subgroup of $W$; the root system $\Phi_{1}$ of $W_{1}$ is a subsystem of $\Phi$ and it follows from $\left[\mathrm{D},(3.3)\right.$ and (3.4)] that $\Phi_{1}^{+}=\Phi^{+} \cap \Phi_{1}$ is a positive system in $\Phi_{1}$. Denote by $\Pi_{1} \subset \Phi_{1}^{+}$the corresponding simple system in $\Phi_{1}$. There is a length function $\ell(w)$ on $W$ which is determined by the simple system $\Pi$ and Corollary (3.4)(ii) of [D] asserts that

(4.1). Each coset $w W_{1}$ of $W_{1}$ in $W$ contains a unique element $\sigma$ with $\ell(\sigma)$ minimal.

Our main result (1.5) may be interpreted in this context as follows.

(4.2) Corollary. Suppose that $W$ is a finite Coxeter group on $V$ with root system $\Phi \subset V$. Let $W_{1}$ be any reflection subgroup of $W$ and let $w \in N_{W}\left(W_{1}\right)$. Then $\sigma \in w W_{1}$ has minimal reflection length in $w W_{1}$ if and only if there exists a simple system $\Pi \subset \Phi$ such that $\sigma$ is the unique element of the coset $w W_{1}$ with $\ell(\sigma)$ minimal (cf. (4.1)). Such elements are all conjugate under $W_{1}$.

Proof. Let $\Pi \subset \Phi$ be a simple system and suppose $\sigma \in w W_{1}$ with $\ell(\sigma)$ minimal. It follows from $[\mathrm{D},(3.4)]$ that $\sigma \Pi_{1} \subset \Phi^{+}$and since $\sigma$ normalises $W_{1}, \sigma \Pi_{1} \subset \Phi_{1}$. So $\sigma \Pi_{1}$ is a simple system in $\Phi_{1}$ which is contained in $\Phi^{+} \cap \Phi_{1}=\Phi_{1}^{+}$, whence $\sigma \Pi_{1}=\Pi_{1}$. We may now apply Theorem (1.5), with $W$ replaced by $W_{1}$ and $\Pi$ by $\Pi_{1}$ to deduce that $n(\sigma)$ is minimal.

If $\sigma^{\prime} \in w W_{1}$ is such that $n\left(\sigma^{\prime}\right)$ is minimal, then by (1.5), $\sigma^{\prime}$ is conjugate under $W_{1}$ to $\sigma$, which proves the remaining assertions.

The following fusion result is an immediate consequence of (4.2). 
(4.3) Corollary. Suppose that $W$ is a finite Coxeter group on $V$ with root system $\Phi \subset V$ and simple system $\Pi \subset \Phi$. Let $W_{1}$ be any reflection subgroup of $W$. If $\sigma \in N_{W}\left(W_{1}\right)$ is the shortest element in $\sigma W_{1}$, then any $W$-conjugate of $\sigma$ which lies in $\sigma W_{1}$, is $W_{1}$-conjugate to $\sigma$.

\section{(4.4) Concluding Remarks.}

(1) The last sentence of the statement (4.2) is stronger than (1.4).

(2) In (4.3), $\sigma$ could equally be assumed to have minimal reflection length in the coset $\sigma W_{1}$. This form of the statement would eliminate specific reference to any simple system $\Pi$.

(3) The statement (4.3) may be reformulated in terms of commutators. For any subgroup $H$ of $W$, write $[\sigma, H]$ for the commutator set $\left\{\sigma^{-1} h^{-1} \sigma h \mid h \in H\right\}$. Then in the the notation of (4.3), $[\sigma, W] \cap W_{1}=\left[\sigma, W_{1}\right]$.

(4) The first part of the statement (4.2) is clearly false if the assumption that $w$ normalises $W_{1}$ is dropped, as may be seen in the example when $W_{1}=<r>$ is generated by a simple reflection. If $s$ is another simple reflection which does not commute with $r$, then the element of $r s W_{1}$ which has minimal length is $r s$, which has reflection length 2. But $r s r$ has reflection length 1.

(5) If $W_{1}$ is a reflection subgroup of the finite Coxeter group $W$ as in (4.2), it is not always true that the elements of minimal reflection length in a coset $w W_{1}$ are conjugate even in $W$, without the assumption that $w$ normalises $W_{1}$. For example if $W$ is a Weyl group of type $B_{4}$, write its elements as monomial matrices with non-zero entries \pm 1 and take $W_{1}$ to be the reflection subgroup (of type $A_{1}^{4}$ ) generated by the reflections

$$
\left[\begin{array}{llll}
0 & 1 & 0 & 0 \\
1 & 0 & 0 & 0 \\
0 & 0 & 1 & 0 \\
0 & 0 & 0 & 1
\end{array}\right],\left[\begin{array}{cccc}
0 & -1 & 0 & 0 \\
-1 & 0 & 0 & 0 \\
0 & 0 & 1 & 0 \\
0 & 0 & 0 & 1
\end{array}\right],\left[\begin{array}{llll}
1 & 0 & 0 & 0 \\
0 & 1 & 0 & 0 \\
0 & 0 & 0 & 1 \\
0 & 0 & 1 & 0
\end{array}\right],\left[\begin{array}{cccc}
1 & 0 & 0 & 0 \\
0 & 1 & 0 & 0 \\
0 & 0 & 0 & -1 \\
0 & 0 & -1 & 0
\end{array}\right] \text {. }
$$

The elements $w=\left[\begin{array}{cccc}1 & 0 & 0 & 0 \\ 0 & 0 & 1 & 0 \\ 0 & -1 & 0 & 0 \\ 0 & 0 & 0 & 1\end{array}\right]$ and $\left[\begin{array}{cccc}1 & 0 & 0 & 0 \\ 0 & 0 & -1 & 0 \\ 0 & -1 & 0 & 0 \\ 0 & 0 & 0 & -1\end{array}\right]$ are in the same coset $w W_{1}$; both have minimal reflection length 2 in the coset, but they have different eigenvalues whence they are not conjugate.

(6) In [L], formulae were given for the number of rational semi-simple orbits of a given "type" (a type is defined by a conjugacy class in $W$ ) in the Lie algebra $\mathfrak{G}$ of $G(G$ as in $\S 1)$ (see, e.g. [L, Theorem (5.6)]). These formulae involve the number of conjugates of $\sigma$ in the coset $w W_{1}$ (notation as in the previous remark). This number is easily seen to be equal to the cardinality of the commutator set $\left[\sigma, W_{1}\right]=\left\{\sigma^{-1} u^{-1} \sigma u \mid u \in W_{1}\right\}$ which in turn is equal to $\left|W_{1} / C_{W_{1}}(\sigma)\right|$, where $C_{W_{1}}(\sigma)$ denotes the set of elements of $W_{1}$ which commute with $\sigma$.

\section{REFERENCES}

[B] N. Bourbaki, Groupes et algèbres de Lie Ch. IV, V, VI, Hermann, Paris 1968

[BT] A. Borel and J. Tits, Groupes réductifs, Publ. Math. IHES 27 (1965), 55-152.

[C1] R.W. Carter, Conjugacy classes in the Weyl groups, Compositio Math. 25 (1972), 1-52

[C2] R.W. Carter, Finite groups of Lie type. Conjugacy classes and complex characters, Pure and Applied Mathematics. A Wiley-Interscience Publication. John Wiley \& Sons, Inc., New York, 1985. 
[D] M. Dyer, Reflection subgroups of Coxeter systems, J. Algebra 135 (1990), 57-73.

[HL] M. Herzog and G.I. Lehrer, A note concerning Coxeter groups and permutations, Proc. MiniConf. Canberra 1975, Springer Lecture Notes 573 (1977), 53-56.

[L] G.I. Lehrer, Rational tori, semisimple orbits and the topology of hyperplane complements, Commentarii Math. Helv. 67 (1992), 226-251.

[St] R. Steinberg, Endomorphisms of linear algebraic groups, Memoirs of the American Mathematical Society, No. 80, American Mathematical Society, Providence, R.I. 1968108 pp. 